Peer Reviewed Paper openaccess

\title{
Hyperspectral imaging for textile sorting in the visible-near infrared range
}

\author{
Carolina Blanch-Perez-del-Notario, ${ }^{\mathrm{a}, *}$ Wouter Saeys ${ }^{\mathrm{b}}$ and Andy Lambrechts ${ }^{\mathrm{c}}$ \\ almec, Kapeldreef 75, 3001, Leuven, Belgium and Division of Mechatronics, Biostatistics and Sensors, KU Leuven, 3001, Leuven, Belgium. \\ E-mail: Carolina.Blanch@imec.be \\ bDivision of Mechatronics, Biostatistics and Sensors, KU Leuven, 3001, Leuven, Belgium. ㄴ https://orcid.org/0000-0002-5849-4301 \\ Imec, Kapeldreef 75, 3001, Leuven, Belgium
}

Recycling of textile materials is becoming important due to the increasing amount of textile waste and its large environmental impact. The Resyntex project aims at dealing with this textile waste by enabling its chemical recycling. To do so, pure textile materials and blends need to be sorted first. In this paper we evaluate the suitability of hyperspectral imaging for pure and blend textile sorting. We also test the discrimination capacity between denim and non-denim textile, since this is required prior to the de-colouration processes. For this purpose, we use a line-scan sensor in the 450-950 nm range, since its cost, compactness and speed characteristics make it suitable for industrial deployment. To deal with the strong colour interference of the textile a hierarchical classification approach is proposed. The results on the available sample set show promising discrimination potential for material discrimination as well as for denim versus non-denim detection.

Keywords: vis-NIR spectral response, textile material, colour classification

\section{Introduction}

The textile sector uses a huge quantity of raw materials and produces a substantial amount of waste. This is partly due to the fact that only a small number of wearable textiles are recycled. Most of these textiles are sent to landfill or incinerated, with a high environmental impact. The European project RESYNTEX ${ }^{1}$ aims to design and develop an industrial symbiosis between the unwearable blends (wool, cotton, synthetic polymers) of garment textile waste and the chemical industry. To enable chemical recycling, sorting of textile material according to material/blend is required first. In this respect hyperspectral imaging has great poten- tial for material discrimination. However, the adoption of hyperspectral imaging by the industry has so far been limited due to the lack of fast, compact and cost-effective hyperspectral cameras with adequate specifications. To bridge the gap between research and industry, Imec has developed a unique hyperspectral sensor concept in which the spectral unit is monolithically integrated on top of a standard CMOS sensor at wafer level. This greatly reduces the cost and improves the compactness and speed of the hyperspectral camera, enabling the adoption of hyperspectral technology by industry. Therefore, we evaluate the use of
Correspondence

C. Blanch-Perez-del-Notario (Carolina.Blanch@imec.be)

Received: 20 February 2019

Revised: 10 September 2019

Accepted: 10 September 2019

Publication: 2 October 2019

doi: 10.1255/jsi.2019.a17

ISSN: 2040-4565
Citation
C. Blanch-Perez-del-Notario, W. Saeys and A. Lambrechts,
"Hyperspectral imaging for textile sorting in the visible-near infrared range",
J. Spectral Imaging 8, a17 (2019). https://doi.org/10.1255/jsi.2019.a17
c 2019 The Authors
This licence permits you to use, share, copy and redistribute the paper in
any medium or any format provided that a full citation to the original
paper in this journal is given, the use is not for commercial purposes and
the paper is not changed in any way. 
the Imec line-scan 150 sensor $^{2}$ providing us with 150 bands in the $450-950 \mathrm{~nm}$ range for the purpose of textile discrimination. Most of the state-of-the-art work on textile discrimination so far has focused on textile sorting in the short-wave infrared (SWIR) range (1000$2500 \mathrm{~nm}$ ). ${ }^{3,4}$ We have explored instead the feasibility for textile discrimination in the visible-near infrared (VNIR) range covered by Imec sensors, since our VNIR cameras allow for cheaper and more compact inspection devices. In addition to this, we have investigated the potential for sorting blue denim textile with respect to other blue cotton textile, since this is a required step for some recycling processes. To our knowledge there is currently no state-of-the-art work on denim discrimination in the VNIR range. In Reference 5, discrimination in SWIR of pure cotton versus denim is addressed and very few samples are considered. In References 6 and 7 the authors focus on discrimination of single textile fibres with indigo dye and this is done with ultraviolet-visible light in either transmittance ${ }^{6}$ or based on fluorescence. ${ }^{7}$

\section{Camera systems}

The imaging system used for all tests in this study is shown in Figure 1, with an Adimec hyperspectral camera and a translation stage where the textile pieces are placed. The imec VNIR line-scan sensor is available in two versions: one acquiring 100 bands in the 600-1000 nm range and another one acquiring 150 bands in the 450-950 nm range. Its spectral unit is integrated in the standard CMOS sensor at wafer level, which reduces its cost and increases the acquisition speed. This way, for standard

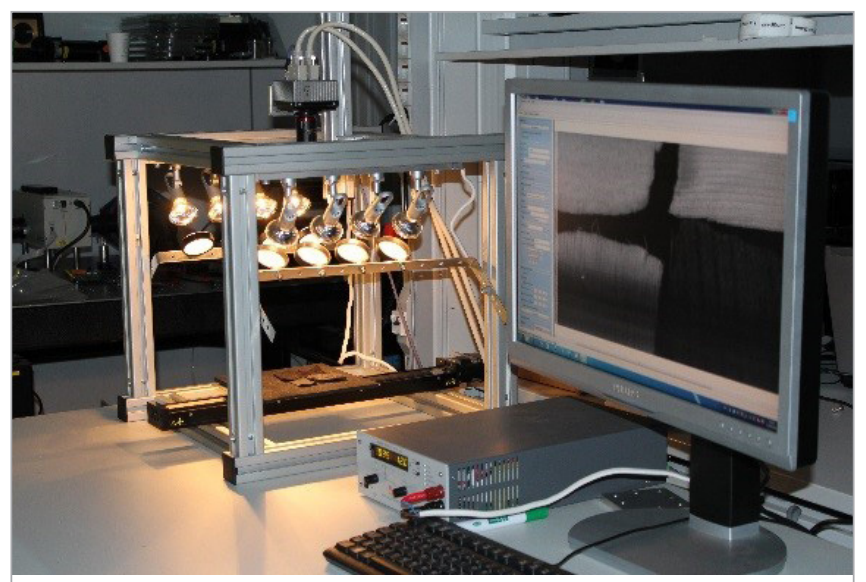

Figure 1. Imec hyperspectral system. halogen-based illumination (325 W) the system can reach a speed of 1080 lines per second.

For the demo setup we use the same type of linescan sensor, but this time integrated in a Ximea camera, ${ }^{8}$ instead of the Adimec camera of the hyperspectral imaging setup of Figure 1 . The main reason for choosing the Ximea camera in the demo setup is that it connects via a USB 3.0 cable to any laptop and does not require a CameraLink interface or frame grabber connection to a desktop PC as the Adimec camera does. This allows an easier development for demo purposes.

The dimensions of our conveyor belt are approximately $11 \times 50 \mathrm{~cm}$, the camera is placed $70 \mathrm{~cm}$ above the conveyor belt, with a $35 \mathrm{~mm}$ lens (Figure 2). The next section explains the hierarchical classification method used for textile sorting based on our hyperspectral imaging setup.

\section{Materials and methods}

This section describes the materials and methods used for the different experimental tests performed. These

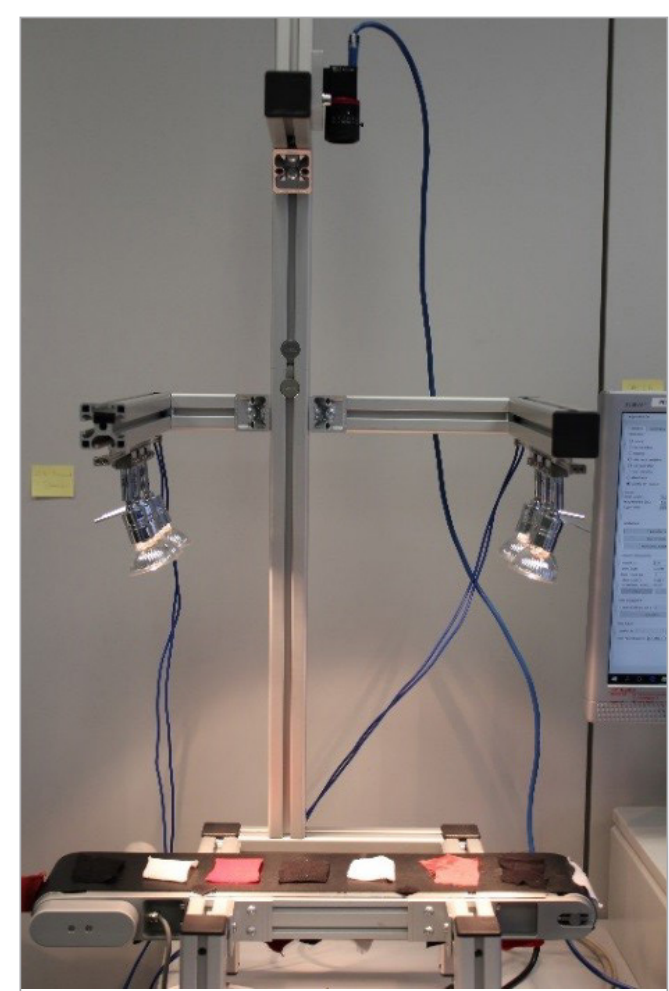

Figure 2. Conveyor belt demo setup. 
Table 1. Summary of tests performed.

\begin{tabular}{|l|l|l|l|}
\hline Experimental test & Camera system & Textile set & Analysis method \\
\hline $\begin{array}{l}\text { Test 1: Reduced textile } \\
\text { set }\end{array}$ & $\begin{array}{l}\text { Adimec } \\
(450-900 \mathrm{~nm})\end{array}$ & $\begin{array}{l}\text { Set of 50 textile pieces of } \\
\text { different materials in four } \\
\text { colours }\end{array}$ & $\begin{array}{l}\text { Colour classification: lab-based and } \\
\text { Material classification: QDC/SVM }\end{array}$ \\
\hline $\begin{array}{l}\text { Test 2: Extended textile } \\
\text { set for one colour } \\
\text { category }\end{array}$ & $\begin{array}{l}\text { Adimec } \\
(600-1000 \mathrm{~nm})\end{array}$ & $\begin{array}{l}\text { Set of } 100 \text { black textile } \\
\text { pieces of different } \\
\text { materials }\end{array}$ & Material classification (SVM) \\
\hline $\begin{array}{l}\text { Test 3: Proof-of- } \\
\text { concept demo }\end{array}$ & $\begin{array}{l}\text { Ximea } \\
(600-1000 \mathrm{~nm})\end{array}$ & $\begin{array}{l}\text { Set of 16 textile pieces in } \\
\text { black, red and white }\end{array}$ & $\begin{array}{l}\text { PCA + QDC for both colour and } \\
\text { material classification }\end{array}$ \\
\hline $\begin{array}{l}\text { Test 4: Denim } \\
\text { discrimination }\end{array}$ & $\begin{array}{l}\text { Adimec } \\
(450-900 \mathrm{~nm})\end{array}$ & $\begin{array}{l}\text { 27 non-denim and 13 } \\
\text { denim textile pieces }\end{array}$ & $\begin{array}{l}\text { QDC classification } \\
\text { Genetic Algorithm for band } \\
\text { selection }\end{array}$ \\
\hline
\end{tabular}

QDC: Quadratic Discriminant Classifier; SVM: support vector machine; PCA: principal component analysis

tests are summarised in Table 1 and further explained in this section.

\section{Test 1: Hierarchical classification for textile material discrimination}

While in the SWIR range the impact of the colour tint on the textile spectra is very low, in the VNIR range the colour impact is very high. This significantly increases the intra-class variability per material type and increases the difficulty for material classification. Figure 3 shows how the average spectra of different colours of $100 \%$ cotton samples varies greatly, indicating, therefore, a strong impact of the colour tint. We display the reflec-

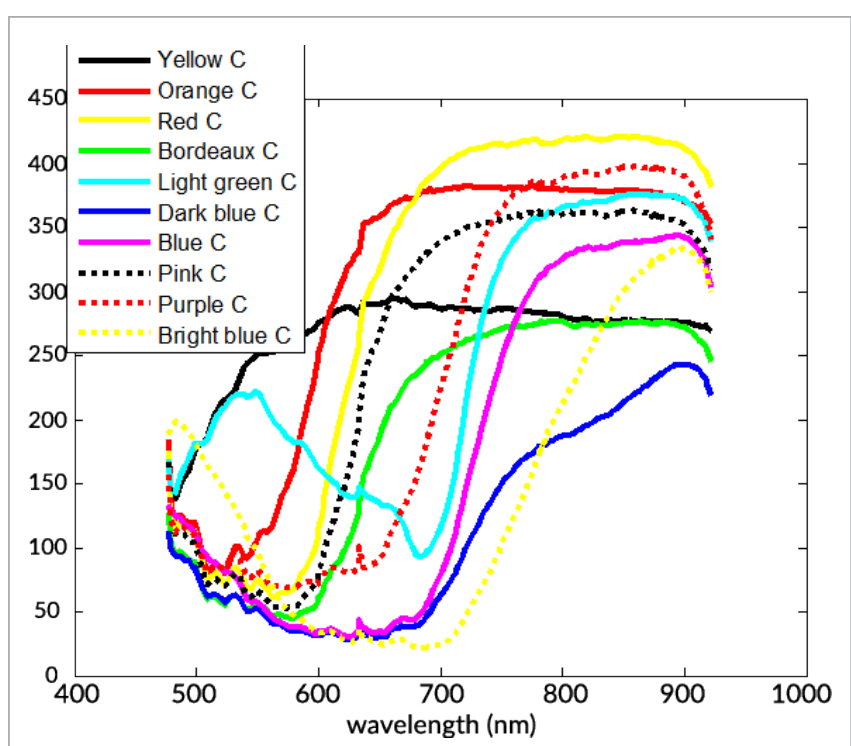

Figure 3. Colour impact on scaled reflectance spectra. tance spectra scaled back to its digital number (2 to the power of 10 bits in this case). Similarly, Figure 4 shows another example of the strong impact of the textile colour on the measured spectra. In this case we show spectra of several textile samples in two colour types: red and blue. For each of the colours, three materials are considered: $100 \%$ cotton, $100 \%$ polyester and $100 \%$ silk. As we can see, the spectra of the same colour and different material have a more similar appearance than spectra of the same material and different colour.

We deal with this colour influence by implementing hierarchical classification, in which colour classification is followed by material classification per colour category. Therefore, we focus on testing mainly four colour categories: black, white, blue and red, for which we have samples available for most of the different materials considered (cotton, polyester, wool, viscose, polyamide, silk, acrylic and cotton blends). All samples are extracted from waste textile garments provided by our partner in the Resyntex project, SOEX. ${ }^{9}$ To facilitate the scan process with our camera system we cut the textile garments received into approximately $5 \times 5 \mathrm{~cm}$ samples with the typical thickness of the sample ranging between one and a few $\mathrm{mm}$. Since some of the textiles are thin, there can be an impact of the background material on the acquired spectrum of the textile sample. To avoid this, we use a black velvet background material of flat and low spectral response over the whole range. At this stage our set of available samples was rather limited ( 50 for all four colours altogether), consisting 


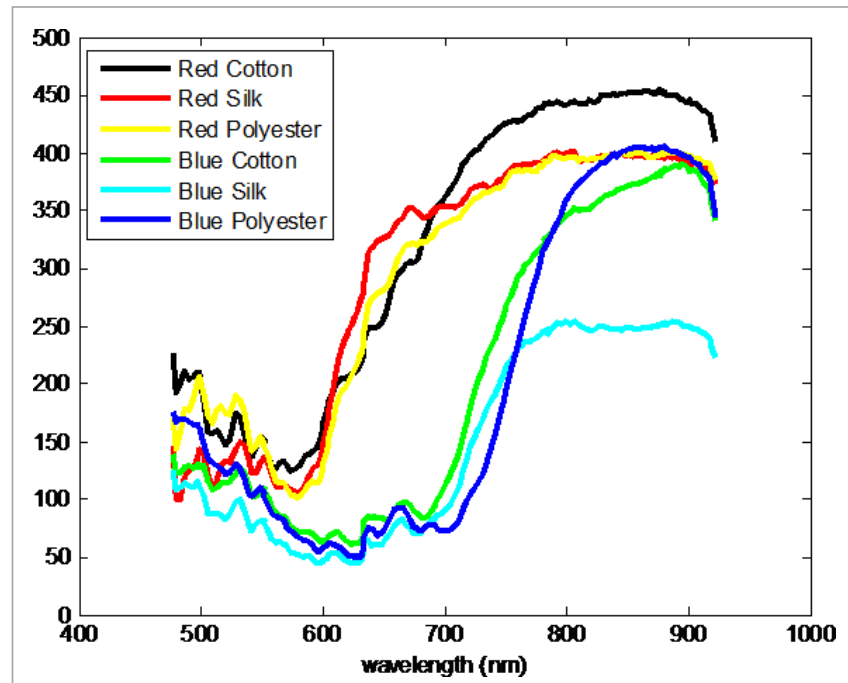

Figure 4. Scaled reflectance with combined material and colour impact.

of one to three samples at most for each colour and material category.

It is not only the colour tint that has a strong impact on the spectra in the $400-1000 \mathrm{~nm}$ range, there is also an impact of the textile material considered in the spectra. This results in the fact that even for identical colour there will be differences in the measured spectra (otherwise no material discrimination would be feasible), this phenomenon is called metamerism ${ }^{10}$ and refers to different spectral signatures showing in the visual domain as the same colour.

Due to this metamerism, it is advisable to approach colour classification by translating the spectra into three colour parameters typically considered for colorimetry: the CIE "L", "a" and "b" parameters. ${ }^{11,12}$ These parameters represent all possible colours since they mimic the way our eyes interpret colour. The three coordinates of CIELAB represent the lightness of the colour ( $L=0$ yields black and $L=100$ indicates diffuse white; specular white may be higher), its position between red/magenta and green ( $a^{*}$, negative values indicate green while positive values indicate red) and its position between yellow and blue ( $b^{*}$, negative values indicate blue and positive values indicate yellow). The $L, a, b$ parameters are computed from the spectral signature as given in the equations in References 11 and 12. With these equations, the L,a,b values can be computed based on the given spectrum, $S$, the illuminant function I, (here assumed to be the D65 standard for average daylight ${ }^{13}$ ) and the CIE observer functions, $x, y, z^{12}$ given

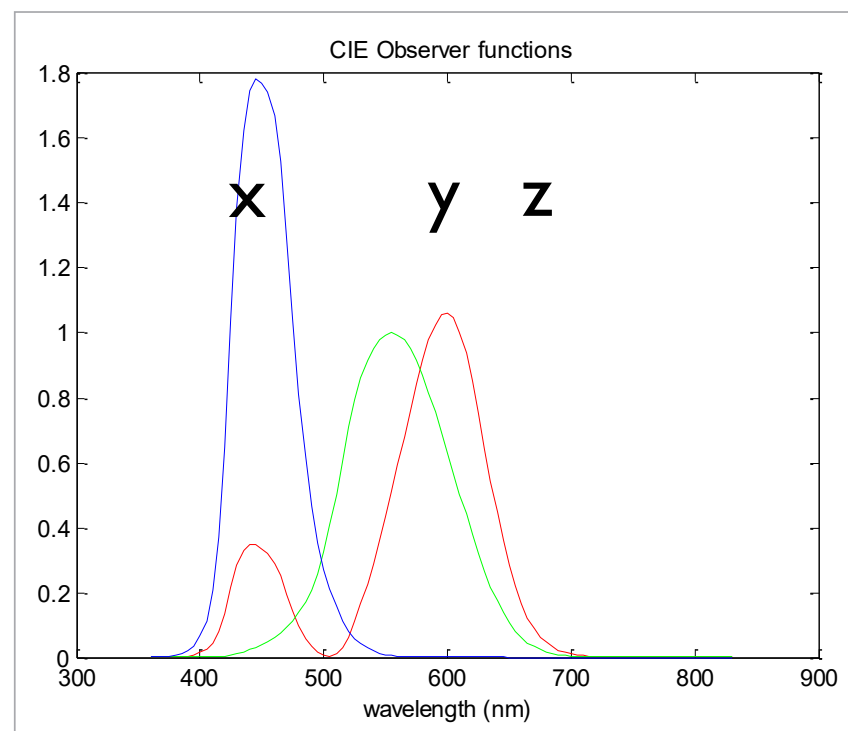

Figure 5. Sensitivity in CIE observer functions.

by Figure 5 corresponding to a $10^{\circ}$ viewing angle. ${ }^{14}$ These observer functions, $x, y$ and $z$ are the numerical description of the chromatic response of the observer, mimicking the tri-stimulus response of the human eye to blue, green and red colours, respectively. Based on the L,a,b values, we can then group colours in the colour space according to pre-defined colour categories. Since we do not have a high variety in sample colour content, we chose 18 colour categories from our training set. The categories selected within the available colour samples are: "Black", "Grey", "White", "Darkblue", "Blue", "Lightblue", "Turquoise", "Green”, "Darkgreen”, "Bordeaux", "Red", "Orange", "Yellow", "Pink", "Fucsia", "Flesh", "Purple", "Darkpurple" and "Beige". The purpose of the selection of this high number of colours is to test the accuracy of our colour classifier. However, a colour classification into a lower basic category may be sufficient to perform material classification in practice.

Colour classification is then performed by means of L,a,b parameter computation of the measured spectrum and selection of the L,a,b value with closest Euclidean distance from the colours available in the library.

To do so we first use a training set of different textile colours to build a library. This training set must contain different colour samples that are representative of all the colour categories identified. The library of Lab values is built by computing the mean Lab value of the textile samples in that category. Finally, to validate this approach, we use a test set with a different set of samples to those used for the training set. We compute the mean Lab 
value for each textile piece in the test set and compare it with the available library Lab values. The one with the closest Euclidean Distance ${ }^{15}$ is selected as the most similar colour (or corresponding colour label). When a colour in the test set is not present in the available colour library, then the most similar colour (in Lab values) will be selected.

After colour classification is made, material classification is then performed for each colour category. From the spectra gathered for each textile sample and colour we use $50 \%$ as the training set for the classifier and the remaining $50 \%$ as the test set. Ground truth is available since we only use materials for which we know the composition. We use the QDC ${ }^{16}$ implemented in the perClass software. ${ }^{17} \mathrm{SVM}^{18}$ also showed similar discrimination capabilities.

\section{Test 2: Extended test for the black colour category}

We have extended a previous material discrimination test for one colour category: black textiles. We now test a set covering almost 100 different black textile pieces coming from a real and representative waste textile sample of around 1 ton of cloth items. In this ton of waste textile, specifically textile garments such as trousers or t-shirts, we found a relative abundance of material, as given in Table 3. This means that, for example, out of the 100 textile samples, 33 of them correspond to pure cotton but only 1 to acrylic. The statistic or relative abundance found in these samples corresponds as well with the ones found in the literature ${ }^{19}$ and can be considered, therefore, representative of typical garment waste. We started testing with black colour items since it is expected to be an abundant colour in the fashion industry. ${ }^{20}$

Table 2. Relative abundance in extended black set.

\begin{tabular}{|l|c|l|l|}
\hline Pure & (\%) & Blend & (\%) \\
\hline Cotton & $33 \%$ & Cotton \& PET & $18 \%$ \\
\hline Viscose & $8 \%$ & PET \& cotton & $6.8 \%$ \\
\hline PET & $4.5 \%$ & Cotton \& viscose & $3.4 \%$ \\
\hline Polyamide & $4.5 \%$ & Viscose \& PA & $2.2 \%$ \\
\hline Wool & $2.2 \%$ & Acrylic \& PA & $2.2 \%$ \\
\hline Acrylic & $1.1 \%$ & Cotton \& PA & $2.2 \%$ \\
\hline Silk & $1.1 \%$ & Acrylic \& wool & $2.2 \%$ \\
\hline Rayon & $1.1 \%$ & Acrylic \& cotton & $1.1 \%$ \\
\hline Linen & $1.1 \%$ & Wool \& PET & $1.1 \%$ \\
\hline
\end{tabular}

The material composition abundance among all our samples is given in percentages in Table 2 .

In this case, due to the increased number of materials, the classifier that performs best is an SVM classifier with radial basis function (RBF) Kernel. ${ }^{21}$ Training samples are based on spectral means of 200-pixel regions of every cloth item. The averaging is done in order to reduce the noise level, reduce the intra-class variability and reduce the SVM training time simultaneously. As in the previous experiment we train with $50 \%$ of the available mean spectra but 12 independent textile samples are also kept for validation (five for cotton, one for viscose, one for cotton \& viscose, one for polyester, one for wool, one for polyamide, two for cotton \& polyester). Since cellulose materials (such as cotton and viscose) are treated together in the recycling process we can group both in a common class.

Note also that cellulose material (cotton \& viscose), both as pure and in a blend (often with polyester), accounts for almost $70 \%$ of the textile waste. Cellulose is therefore, in pure form and in blend, one of the most economically relevant materials for textile recycling processes. The rest of the pure materials such as polyester, polyamide, wool and silk account for around $15 \%$ of the total waste and they are as well quite relevant materials for further recycling. Other minor blends (e.g. acrylic and polyamide blends with any other material) have very low presence and are discarded from this study due to their low relevance for the recycling processes. Blends of polyamide and acrylic are more difficult to process for recycling and in addition, are not economically viable due to their very low abundance. We label all these materials in the "Other material" category.

\section{Test 3: Proof-of-concept demo}

We show a proof-of-concept demo of the hierarchical classification scheme, composed of colour classification first and, second, material classification per colour. To do so, we select a subset of the previously mentioned samples and place them on a small conveyor belt. Due to the limited space on the conveyor belt we restrict ourselves to a subset of 16 of the previous samples. Figure 6 shows the materials used in the demo and their composition.

As already explained, textile sorting in the VNIR range requires a hierarchical classification approach to deal with colour interference in the VNIR spectrum: 


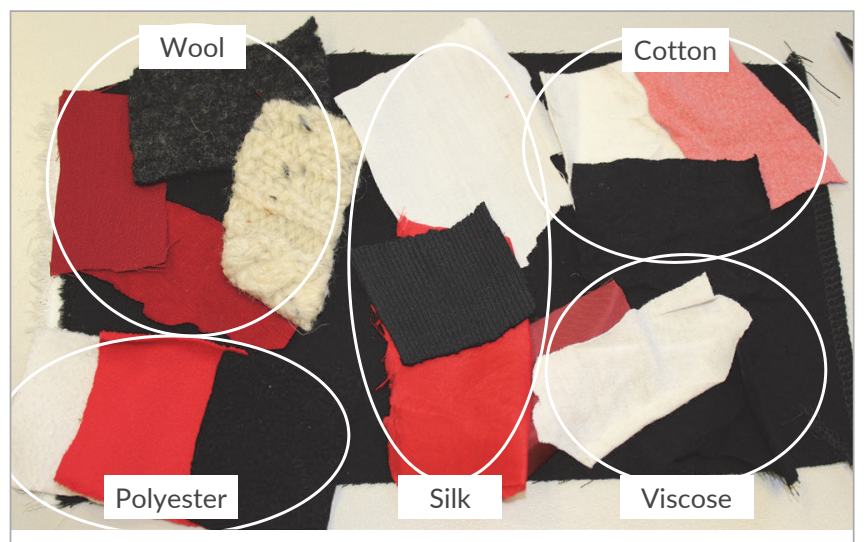

Figure 6. Textile materials for demo set.

colour classification to establish the textile colour category,

material classification within each colour category.

We add a first additional step which consists of classifying background versus objects. This allows us to later do some filtering or majority count vote at the object level, which corrects a few misclassifications on individual pixels. After object discrimination, colour classification is performed on the object pixel. Depending on the colour label obtained, the corresponding material classifier per colour category is used. The final result is a classifier label indicating a material type regardless of the textile colour.

Depending on the classification label attached to each pixel of the image a different colour is shown on the screen. The colours used per classified label are green for polyester, blue for wool, pink for silk, yellow for cotton and red for viscose.

Both Lab-based classifiers for colour classification and QDC and SVM classifiers trained and presented in the previous section for material classification could have been used to classify the textiles in the demo. However, to meet real-time classification in our demo we need to adapt our classifier implementation. To do this, we use already available classification units in the perClass analysis software. Since Lab value computation is not provided, we train a QDC classifier to discriminate between the red, white and black colour groups, regardless of the material. Discrimination between these three colours can be achieved by a QDC classifier from the spectral values. However, for discrimination of a high number of colours, classification based on Lab computation from the spectra would be more suitable and robust. Moreover, to meet real-time constraints we use PCA as a preprocessing step for both colour and material classifiers. In our demo each classifier module consists in PCA extraction followed by a QDC, ${ }^{16}$ as implemented in the perClass hyperspectral analysis software. ${ }^{17} \mathrm{PCA}^{22}$ is used as a method to reduce the dimensionality of the input. This is mainly required for speed performance requirements. When the classification module is implemented on a reduced dimensionality the classification speed increases, which is desirable for real-time classification. Training of the classification algorithms is done by means of perClass software. After training, a combined executable file of the classifier (PCA + QDC) is created so that it can be used for the demo application. This makes it easier to integrate the classifier code in our GUI. In addition to the perClass classifier algorithms, an object level-based majority count vote is implemented. This means that for each object (piece of textile) identified, all pixels are labelled with the class of the majority of the pixels in the object.

\section{Test 4: Denim discrimination}

Denim textiles are mainly composed of cellulose and constitute around $15 \%$ of the total waste stream. Therefore, denim processing is very important for the recycling industry.

The most common denim textile is indigo denim, in which the warp thread is dyed, while the weft thread is left white. In the textile recycling process de-colouration of textiles is generally required prior to any further chemical processing of the raw textile components (cotton/ polyester/wool...). In this respect, since the indigo dye used in denim materials requires a specific de-colouration process, the separation of blue denim material prior to de-colouration becomes a useful step. For this purpose, we tested the discrimination possibilities of VNIR hyperspectral imaging to separate blue denim with respect to any other blue textile types. Note that in terms of composition, both denim and other cotton/polyester blends can be very similar.

To test denim discrimination, we used 27 non-denim textiles and 13 denim textiles. Non-denim textiles are mostly polyester and cotton blends, some very similar in composition to denim. As in previous tests the samples originate from textile garments (trousers mainly) and are cut to roughly $5 \times 5 \mathrm{~cm}$. Additionally, a black velvet material with low and flat spectral response is used as background to minimise spectral interference from the background. We consider four classes: "Background", "Denim", "Other" (i.e. blue textile but non-denim) and 


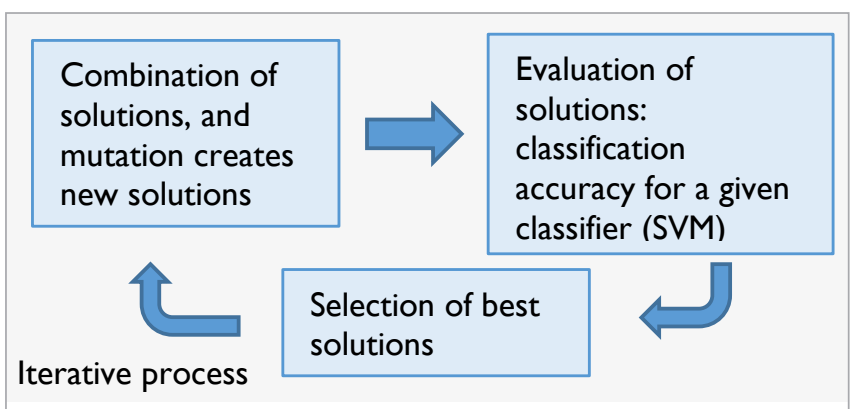

Figure 7. Schematics of a Genetic Algorithm.

"Paper" (label attached to our textile samples). For denim discrimination we train a QDC classifier, since this classifier has shown good performance in previous textile discrimination tests as compared to linear classifiers, with 10,000-pixel spectra per class, out of which $50 \%$ are used for training and the other 50\% for testing. In addition, we use two pieces of denim textile and three pieces of non-denim as independent validation samples. For this purpose, they are kept out of the training set.

To find the most discriminative bands in our wavelength range we use a Genetic Algorithm (GA) ${ }^{23}$ in combination with our classifier. The aim is to find a near-optimal number of reduced bands that still provide high accuracy classification.

The behaviour of the GA is summarised here and in Figure 7:

For a desired number of subset bands/wavelengths (e.g. three) an initial "population" of individuals is created. Each "individual" consists of a specific set of random band selections, e.g. $630 \mathrm{~nm}, 770 \mathrm{~nm}, 900 \mathrm{~nm}$. An individual with a uniform selection is also included in the initial population.

For each individual solution (band selection) the fitness function of the GA is computed as the mean classification accuracy obtained for that band subset on a fixed training and testing set.

-The GA iterates for a given number of iterations the best "individual" or band subset is kept.

\section{Results and discussions}

This section discusses the results obtained in the different tests described in previous section.

\section{Test 1: Hierarchical classification for material discrimination}

Table 3 shows an example of colour classification results when applied on a test set of wool materials with a specific Lab value (left column) who gets assigned to the closest Lab value in the library (right column).

The colour classification results are generally good. Only a few colours that are not so well represented in the current library show slight deviations from the actual colour and the colour label attached by the classifier. This is due to the limited number of categories considered so far. This way, for instance "light green" wool material ends up classified as "beige" (see Table 3). Extending the colour library to include some lighter shades of green and blue would increase the accuracy of colour classification in this case.

For each textile colour category, material classification is performed. Figure 8 shows the material classification results for a "red" set of materials.

While at pixel level there are few misclassifications, at object level the classification is $100 \%$ accurate. Similar results are obtained for other colour categories tested and summarised in Table 4 where the classification accuracy per material and colour set is indicated.

Table 3. Colour classification based on Lab values.

\begin{tabular}{|l|l|}
\hline Wool "real colour" (L,a,b) & Classifier label (L,a,b) \\
\hline Black (29.0, -8.0, -7.4) & Black $(34.5,-12.2,-12.8)$ \\
\hline Red (58.2, 14.3, -17.2) & Red $(54.2,16.9,-12.9)$ \\
\hline Light Green (60.4.-6.0,-2.2) & Beige $(69.5,-5.9,-4.1)$ \\
\hline Beige (63.0, -0.7, -4.2) & Beige $(69.5,-5.9,-4.1)$ \\
\hline Blue (40.9, -8.1, -10.8) & Dark blue (43.1,-7,-10.2) \\
\hline
\end{tabular}



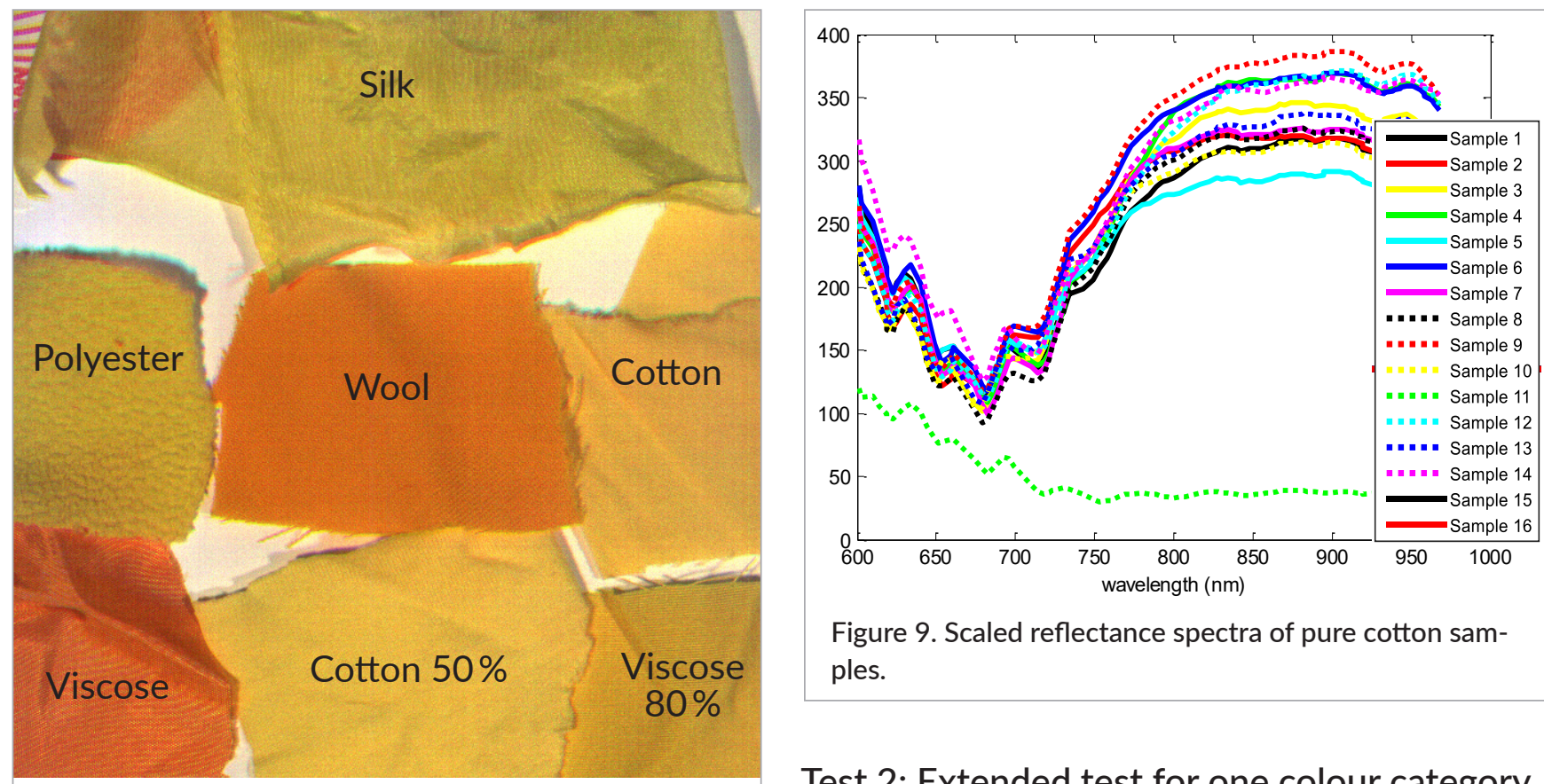

Figure 9. Scaled reflectance spectra of pure cotton samples.

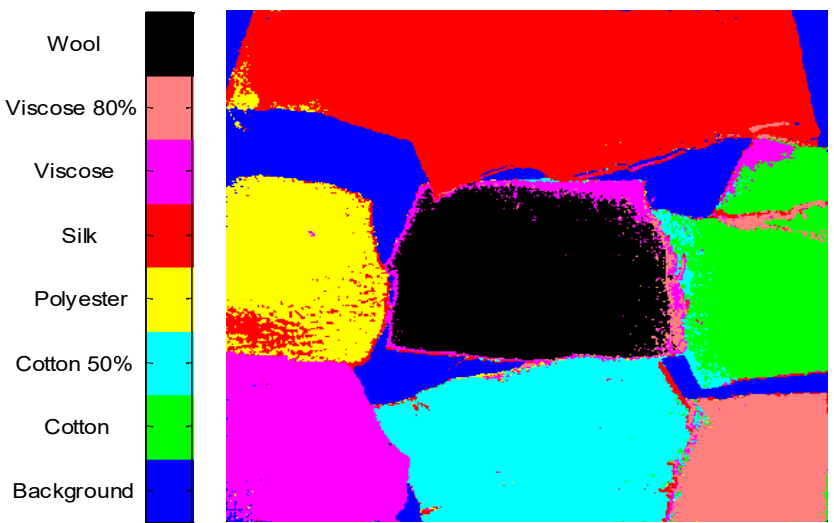

Figure 8. False colour image of red textiles (above) and corresponding classified image (below).

\section{Test 2: Extended test for one colour category}

When inspecting the spectral signature of different black materials, we first notice that some pure and blend materials have an unusual and very low reflectance over the whole VNIR range. Figure 9 shows such an example where the spectral signature of multiple black cotton samples is compared. Note that cotton item 11 has a distinctively low reflectance spectrum as compared to all other black cotton samples. We observe this phenomenon in less than $10 \%$ of the samples of cotton/viscose/ cotton blend. The reason for this different signature is not known, although it is also found in the literature and treated as outlier. ${ }^{24}$ It is not due to the impact of the background underneath, but one possible explanation

Table 4. Material pixel classification accuracy per colour.

\begin{tabular}{|l|c|c|c|c|}
\hline Colour set & Black & White & Blue & Red \\
\hline $100 \%$ Cotton & $100 \%$ & $93 \%$ & $92.5 \%$ & $91.5 \%$ \\
\hline $100 \%$ PET & $100 \%$ & $95 \%$ & $100 \%$ & $95 \%$ \\
\hline $100 \%$ Wool & $100 \%$ & $100 \%$ & $90 \%$ & $95 \%$ \\
\hline $100 \%$ Viscose & $97.5 \%$ & $100 \%$ & - & $92.5 \%$ \\
\hline $100 \%$ Polyamide & $100 \%$ & $100 \%$ & $75 \%$ & $100 \%$ \\
\hline $100 \%$ Silk & $100 \%$ & $100 \%$ & $90 \%$ & $100 \%$ \\
\hline $100 \%$ Acrylic & - & $90 \%$ & $100 \%$ & $100 \%$ \\
\hline $80 \%$ Cotton & - & $60 \%$ & $88 \%$ & $70 \%$ \\
\hline $60 \%$ Cotton & $100 \%$ & $90 \%$ & - & $85 \%$ \\
\hline
\end{tabular}


could be the dye applied to the cloth item. For the purpose of the analysis we consider these materials as outliers that are identified and discarded prior to material classification.

Since cellulose materials (such as cotton and viscose) are treated together in the recycling process, we can group both in a common class. The trained classifier results in a $100 \%$ accuracy of classification for the textile samples from the training set.

To test the generalisation capabilities of the classifier we use an independent test set of samples and focus on the most relevant materials for the recycling processes, namely cellulose, polyester, cellulose blends, polyamide and wool. We, therefore, use an independent test set containing the following materials and number of cloth items: five cotton items, one viscose, one polyester, one polyamide, one wool and three cotton blends (one cotton/viscose, one cotton/PET and one PET/cotton). The classification accuracy on the independent test set is shown in Table 5.

As we can see, the classification accuracy of most of the samples is reasonably high, especially pure samples. However, the two cotton and polyester blends are misclassified. The cotton \& PET blend spectral averages get misclassified as either pure cotton or pure PET, which is at least consistent with the blend content. The "PET \& Cotton" blend gets misclassified as cotton. The intra-class variability of cotton $\&$ polyester blends may require a higher number of samples within the training set for the classifier to achieve good generalisation capabilities.

We leave for future work the discrimination within extended sample sets in other colour categories (white, blue, grey...) where a more robust discrimination may be feasible than for black colour items.

In addition, knowledge of the chemical recycling process of the different materials would help us to tune the clas-

Table 5. Classification accuracy on test set.

\begin{tabular}{|l|l|}
\hline Test set material & Accuracy (\%) \\
\hline Cotton/viscose & $68 \%$ \\
\hline PET & $70 \%$ \\
\hline Polyamide & $100 \%$ \\
\hline Wool & $100 \%$ \\
\hline Cotton \& PET & $\begin{array}{l}\text { Misclassified 50\% as cotton } \\
\text { and 40\% PET }\end{array}$ \\
\hline PET \& cotton & Misclassified as cotton \\
\hline
\end{tabular}

sification scheme. For instance, we know that viscose and cotton are treated together as cellulose material, which, therefore, can be grouped in the same class. Cotton blends can also be added to the cellulose stream but are initially sorted to estimate the process yield better. Moreover, we know that in the cellulose processing the presence of other textile types such as polyester or cotton blends does not jeopardise the operation, therefore misclassifications of other materials into those is not critical, only affecting the yield estimation slightly. Similarly, the processing of proteins from wool or silk is not affected greatly by other materials. On the contrary, the processing of Polyester or Polyamide requires a pure flow with no other textiles present. In general, acrylics are not processed but should be removed from the previous flows. Although we can expect lower robustness in the VNIR range $(400-1000 \mathrm{~nm})$ with respect to the SWIR range $(1000-2500 \mathrm{~nm})$, the VNIR range is still a relevant one since it allows for compact and faster inspection methods. Moreover, we believe it provides better discrimination capabilities than SWIR for denim textiles, as we will explain in the following sections.
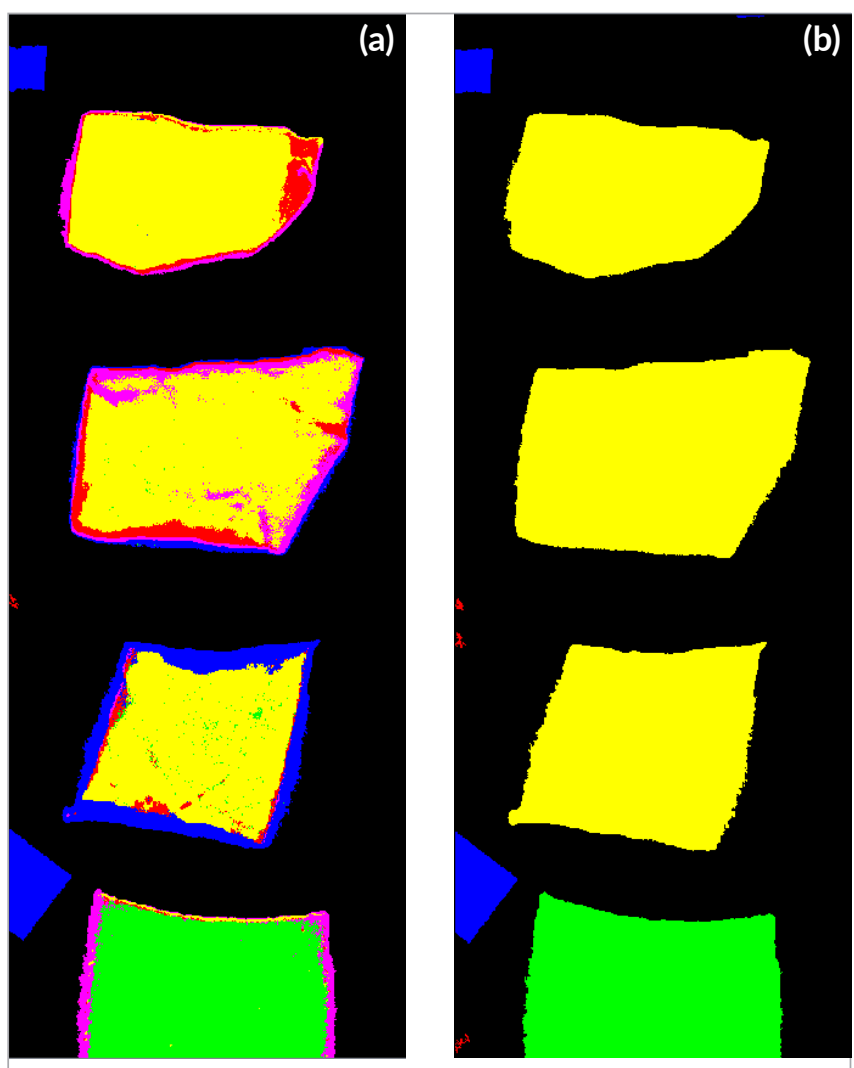

Figure 10. Classification output at pixel level (a) and after object level majority vote (b). 


\section{Test 3: Proof-of-concept conveyor belt demo}

The effect of majority count vote at object level can be seen in Figure 10a and b. This step cleans small misclassifications present in a few pixels (usually at borders and creases) as seen in the screen shots of Figure 10a and b. With the additionally implemented object level majority vote the accuracy becomes $100 \%$ on each textile. Without this additional step the accuracy is somewhere above $90 \%$ of pixels with correct classification on each textile.

The classification speed of our current demo implementation is around $8 \mathrm{cms}^{-1}$. This is fast-enough to appreciate the textile pieces moving rapidly on the conveyor belt while still being able to visualise and control the classified output comfortably.

However, there is still quite some room for parallelisation and memory efficient optimisations. This would lead to considerably higher conveyor and classification speeds since our camera and conveyor belt system can easily deal with up to $80 \mathrm{~cm} \mathrm{~s}^{-1}\left(0.8 \mathrm{~m} \mathrm{~s}^{-1}\right)$, which would be enough for processing one cloth item per second.

The initial pilot system in the Resyntex project expects to reach speeds of $100 \mathrm{~kg} \mathrm{~h}^{-1}$ of processed textiles. This

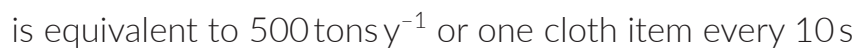
(assuming a $300 \mathrm{~g}$ item on average). In the system under preparation each textile item will be processed separately and, according to the acquired spectra, sorted in a corresponding basket by an air-separator system.

\section{Test 4: Denim versus non-denim discrimination}

The tests performed to assess the discrimination capacity of the VNIR range for blue denim textiles versus all other non-denim blue textiles show that accurate discrimination is feasible. Non-denim textiles are mostly polyester and cotton blends, some very similar in composition to denim. Table 6 shows the pixel classification accuracy obtained in the 470-900 nm range. We train the classifier to discriminate the background and paper label for better visualisation of the classified image but show here the accuracy of the relevant classes of denim and nondenim ("Other textile class").

Table 6. Discrimination of denim vs non-denim.

\begin{tabular}{|l|c|}
\hline Test set material & Accuracy (\%) \\
\hline Blue denim & $98 \%$ \\
\hline Other (blue non-denim) & $97 \%$ \\
\hline
\end{tabular}

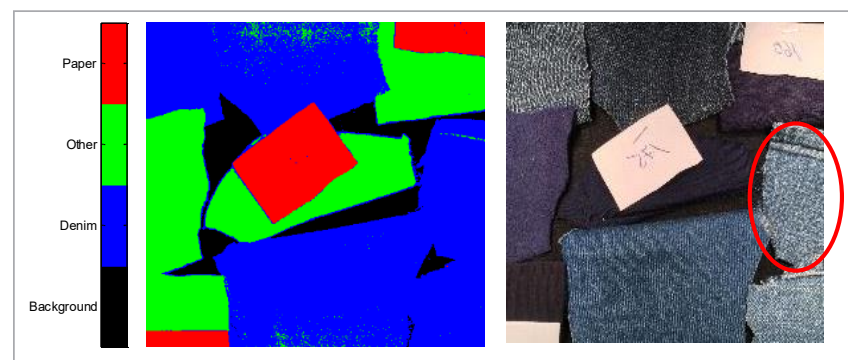

Figure 11. Classified image for denim/non-denim mix.

All five textiles in our independent set (three nondenim and two denim) are accurately classified for over $90 \%$ of the pixels.

An example of output of a classified textile image is shown in Figure 11 with the independent validation denim sample highlighted. Accurate discrimination of denim versus non-denim textiles can be seen, even in cases where the colour tint is very similar.

The band relevance study performed with a Genetic Algorithm results in a selected subset of three bands (624, 696 and $884 \mathrm{~nm}$ ) for which a discrimination accuracy of $90 \%$ can be obtained for both our denim and non-denim set of blue textiles. Note that the wavelengths found to be most discriminative do not belong to the blue range of the spectra (450-595 nm), but closer to the visible range limit (700 nm and almost $900 \mathrm{~nm}$ ) and one band in the red range of the spectrum $(\sim 624 \mathrm{~nm})$.

Higher spectral ranges, such as the SWIR from $1000 \mathrm{~nm}$ to $1700 \mathrm{~nm}$ or $2500 \mathrm{~nm}$ are more agnostic of the colour influence. This has the advantage of suffering lower or no interference from the textile colour and being, therefore, more robust for material identification regardless of the colour. However, in the case of blue denim discrimination this is a disadvantage, since the chemical composition of denim is very similar to those of non-denim textiles: namely a cotton and polyester blend where cotton is in higher percentage. This, together with the good discrimination achieved in our tests, makes us believe that the VNIR range is more useful for the step of denim textile discrimination prior to textile recycling processes.

\section{Conclusions}

The feasibility study performed over a variety of pure textile materials (cotton, viscose, polyester, wool, silk and polyamides) and blends (cotton and polyester, viscose 
and polyester) is promising and seems to indicate that material discrimination can be performed by means of hyperspectral imaging in the VNIR range. In order to guarantee a robust sorting system for all textiles varieties we need to include a more extensive sample set in the training phase. In the same spectral range, we have also assessed that good discrimination between blue denim and blue non-denim textiles can be made, which is a required step prior to textile de-colouration.

Being able to sort textiles in the VNIR range brings many advantages since it offers higher spatial resolution, cheaper and more compact cameras than the traditional SWIR range. Moreover, the VNIR range enables sorting of blue denim, an abundant and relevant component of textile waste that could be difficult to sort in the SWIR range.

\section{Acknowledgements}

This research has been financed by EU project Resyntex from the European Union's Horizon 2020 research and innovation programme under grant agreement No. 641942.

\section{References}

1. http://www.resyntex.eu/

2. P. Gonzalez, K. Tack, B. Geelen, B. Masschelein, W. Charle, B. Vereecke and A. Lambrechts, "A novel CMOS-compatible, monolithically integrated line-scan hyperspectral imager covering the VISNIR", Proc. SPIE 9855, 98550N (2016). https://doi. org/10.1117/12.2230726

3. https://ec.europa.eu/environment/ecoinnovation/ projects/en/projects/t4t

4. http://www.valvan.com/products/equipment-forused-clothing-wipers/sorting-equipment/fibersort/

5. J.S. Yeom, Textile Fingerprinting for Dismount Analysis in the Visible, Near, and Shortwave Infrared Domain. Thesis, Department of the Air Force Air University.

6. S. Suzuki, Y. Suzuki, H. Ohta, R. Sugita and Y. Marumo, "Microspectrophotometric discrimina- tion of single fibers dyed by indigo and its derivatives using ultraviolet-visible transmittance spectra", Science \& Justice 41, 107-111 (2001). https://doi. org/10.1016/S1355-0306(01)71861-8

7. S.L. Morgan, A.A. Nieuwland, C.R. Mubarak, J.E. Hendrix, E.M. Enlow and B.J. Vasser, "Forensic discrimination of dyed textile fibers using UV-VIS and Fluorescence Microspectrophotometry", Proceedings of the European Fibres Group, Prague (2004).

8. https://www.ximea.com/en/products/xilab-application-specific-oem-custom/hyperspectral-camerasbased-on-usb3-xispec

9. https://www.soex.de/en/recycling-germany/

10. https://en.wikipedia.org/wiki/Metamerism_(color)

11. https://en.wikipedia.org/wiki/Lab_color_space

12. https://en.wikipedia.org/wiki/CIE_1931_color_space

13. files.cie.co.at/204.xls (D65 illuminant)

14. https://support.hunterlab.com/hc/en-us/ articles/203420099-CIE-Standard-Observers-andcalculation-of-CIE-X-Y-Z-color-values-AN-1002b

15. https://en.wikipedia.org/wiki/Euclidean_distance

16. http://www.37steps.com/prhtml/prtools/qdc.html

17. http://perclass.com/perclass-toolbox/product

18. http://www.csie.ntu.edu.tw/ cjlin/papers/guide/ guide.pdf

19. Étude de Caractérisation des TLC (Textiles D’habillement, Linge de Maison et Chaussures) Usagés Entrant en Centres de Tri ainsi que des Déchets Ultimes Résultant du Tri (2014).

20. https://qz.com/quartzy/1194798/only-black-is-thenew-black-a-cultural-history-of-fashions-favoriteshade/

21. http://doc.perclass.com/perClass_Toolbox/guide/ classifiers/svm.html

22. L. Smith, A Tutorial on Principal Component Analysis (2002). http://www.cs.otago.ac.nz/cosc453/student tutorials/principal_components.pdf

23. https://en.wikipedia.org/wiki/Genetic_algorithm

24. T. Haran, Short-Wave Infrared Diffuse Reflectance of Textile Materials. Thesis from Georgia State University (2008). 